where $\lambda^{\prime}$ is wave-length of light in liquid, $\theta$ is angle of scattering $(\theta=0$ for transmitted light), $x=\frac{2 \pi D^{*}}{\lambda^{\prime}} \sin \frac{\theta}{2}, D^{*}$ being the characteristic dimension, $D$ is diameter of the sphere and $L$ is length of the rod.

In the early stages of the precipitation, the experimental points fall on the theoretical line associated with the spherical model. In the later stages the plot shows many of the characteristics of scattering expected of rod-like particles. This would be expected if the sample was a mixture of small spheres and linear aggregates of spherical particles.

Work is proceeding in order to elucidate the problem further.

Chemistry Department, University,

Birmingham 15. March 19.

1 Fessler, J. H., Nature, 117, 439 (1956).

${ }^{2}$ Debye, P., J. Phys. and Coll. Chem., 51, 18 (1947).

\section{Latin-Square Designs with Individual Gradients in One Direction}

THE efficacy of the Latin-square design ${ }^{1}$ in eliminating two-dimensional variation from treatment comparisons, particularly in field experiments, has long been established. Kendall ${ }^{2}$ states that horizontal and vertical gradients at least are removed from the comparisons, implying, of course, that the three degrees of freedom for columns in a $4 \times 4$ Latin square, for example, can be regarded as eliminating linear, quadratic and cubic components in one direction. The mathematical model for the analysis can be regarded equivalently as representing the background of the experimental material against which the experiment is conducted as a curved sheet, the curvatures in vertical planes parallel to the columns (rows) being equal for plots in one row (column). In some situations, however, the changes in the experimental material may be such that different curvatures are more realistic, and on the assumption that the more closely the imposed model represents the underlying phenomena, the greater the achievable accuracy, such alternatives merit consideration.

As usual, a completely general expression of the background material introduces difficulties because of the relation between the number of parameters estimated and the number of degrees of freedom for error, so that some compromise guided by a priori knowledge is required, and only the simplest cases have so far been studied.

In one case, for which applications can immediately be envisaged (see below), it is assumed that columns (say) are discrete and that variation within them may be represented in terms of individual linear regressions. The mathematical model used therefore is :

$$
y_{i j}=\mu+x_{j}+\beta_{j} a_{i j}+\theta_{i}+e_{i j}
$$

where $y_{i j}$ is the observed value for the experimental unit of the ith treatment in the $j$ th column; $\mu$ is the general mean; $x_{j}$ and $\beta_{j}$ are the location and slope parameters for the linear variation in the $j$ th column; $a_{i j}$ is the discrete row constant for the $i$ th treatment in the $j$ th row measured from a zero mean at the centre of the rows, and it is convenient to take

$$
\sum_{i=1}^{n} a^{2} i j=\sum_{j=1}^{n} a_{i j}^{2}=n ;
$$

$\theta_{i}$ is the $i$ th treatment parameter.

As usual, it is assumed that the unexplained variate $e_{i j}$ is distributed normally and homoscedastically with variance $\sigma^{2}$ about a zero mean.

Unbiased estimates of treatment comparisons can readily be obtained, and the variance-covariance matrix for an $n \times n$ Latin square is

$$
-n\left[\mathbf{A A ^ { \prime }}-n^{2}\right]^{-1}
$$

where $[\mathbf{A}]$ is the matrix $\left[a_{i j}\right]$.

The linearly declining portions of the lactation curves of dairy cattle and goats form the backgrounds for numerous experiments, in some of which treatment after-effects are absent or sufficiently small to permit the application of the preceding analysis. The corresponding analysis of variance on the left in Table 1 is compared with the conventional analysis of a randomization imposed on the lactation curves of four Guernsey cows.

Table 1. Analyshs of Variance of Milk yiblds (LB./Whek)

\begin{tabular}{|c|c|c|c|c|}
\hline \multirow{2}{*}{ diradien } & & \multicolumn{3}{|c|}{ Conven } \\
\hline & m.s. & & d.f. & m.s. \\
\hline ows & $8,525 \cdot 4518$ & Cows & 3 & $8,525 \cdot 4518$ \\
\hline ng treatments & $553 \cdot 3383$ & Weeks & 3 & $398 \cdot 7018$ \\
\hline or reg. & $35 \cdot 5477$ & re & 3 & $86 \cdot 0976$ \\
\hline $\begin{array}{l}\text { Unexplained varia- } \\
\text { tion }\end{array}$ & $39 \cdot 5352$ & $\begin{array}{l}\text { Unexplained } \\
\text { variation }\end{array}$ & 6 & $177 \cdot 2123$ \\
\hline
\end{tabular}
Gradient YIELDS (IB./W

The treatment estimates for the two methods are :

Gradient analysis Conventional analysis

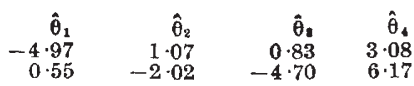

The variance - covariance matrix for the design used is :

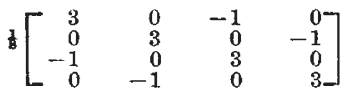

so that the variance estimate for differences between two treatments is either 39.54 or 29.65 ; comparison with the corresponding estimate, 88.61, obtained from the conventional analysis shows that in this case a substantial increase in the amount of information has been achieved. The greatest increases in information may be expected when large differences exist between the individual regression coefficients, a situation more likely to occur in animal than in crop experiments, and the gradient analysis may be especially useful when animals from a small herd cannot easily be matched for stage of lactation.

A. detailed paper, on the methods discussed here and on the general case allowing for higher-order individual regression terms, is in preparation.

Section of Statistics,

\section{P. Cox}

National Institute for Research in Dairying, University of Reading. Feb. 10.

1 Yates, F., Emp. J. Exp. Agric., 1, 235 (1933).

2 Kendall, M. G.. "The Advanced Theory of Statistics", 2 (Griffn, 1946). 